\title{
Phosphorylated chitosan hydrogels inducing osteogenic differentiation of osteoblasts via JNK and p38 signaling pathways
}

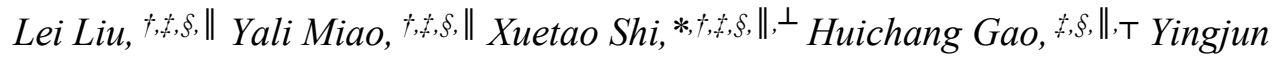

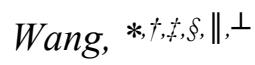

${ }^{\dagger}$ School of Material Science and Engineering, South China University of Technology, Guangzhou 510640, PR China

National Engineering Research Center for Tissue Restoration and Reconstruction, South China University of Technology, Guangzhou 510006, P. R. China

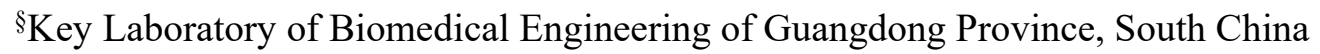
University of Technology, Guangzhou 510006, P. R. China

$\|_{\text {Key Laboratory of Biomedical Materials and Engineering of the Ministry of }}$ Education, South China University of Technology, Guangzhou 510006, P. R. China

${ }^{\perp}$ Guangzhou Regenerative Medicine and Health Guangdong Laboratory, 510005, Guangzhou, China

TSchool of Medicine, South China University of Technology, Guangzhou 510640, PR China

*Email: shxt@,scut.edu.cn (X. S.).

*Email: imwangyj@163.com (Y.W.). 
Table S1. Primer sequences used for real-time PCR.

\begin{tabular}{cl}
\hline Gene name & \multicolumn{1}{c}{ Primer sequence } \\
\hline \multirow{2}{*}{ GAPDH } & 5'-GCACCGTCAAGGCTGAGAAC-3' \\
& 5'-TGGTGAAGACGCCAGTGGA-3' \\
RUNX2 & 5'-CACTGGCGCTGCAACAAGA-3' \\
& 5'-CATTCCGGAGCTCAGCAGAATAA-3' \\
ALP & 5'-CATGCTGAGTGACACAGACAAGAA-3' \\
& 5'-ACAGCAGACTGCGCCTGGTA-3' \\
COL1 & 5'-GCTTGGTCCACTTGCTTGAAGA-3' \\
& 5'-GAGCATTGCCTTTGATTGCTG-3' \\
OCN & 5'-TGTGGAGGGTTGTGGGTGT-3' \\
& 5'-TCAACTGGGGTGGGGTTTT-3' \\
OPN & 5'-GATGAATCTGATGAACTGGTCACTG-3' \\
& 5'-GGTGATGTCCTCGTCTGTAGCA-3' \\
\hline
\end{tabular}

Table S2. Feed ratio and phosphocreatine content of MAC-PCr.

\begin{tabular}{cccccc}
\hline Sample & MAC & PCr & EDC & NHS & PCr Content \\
& $(\mathbf{g})$ & $(\mathbf{g})$ & $(\mathbf{g})$ & $(\mathbf{g})$ & $\mathbf{m g} / \mathbf{g}$ \\
\hline MAC & - & - & - & - & - \\
M-LPCr & 1 & 0.04 & 0.036 & 0.02 & $14.70 \pm 1.22$ \\
M-MPCr & 1 & 0.2 & 0.18 & 0.1 & $43.81 \pm 0.82$ \\
M-HPCr & 1 & 1 & 0.9 & 0.5 & $197.53 \pm 13.08$ \\
\hline
\end{tabular}




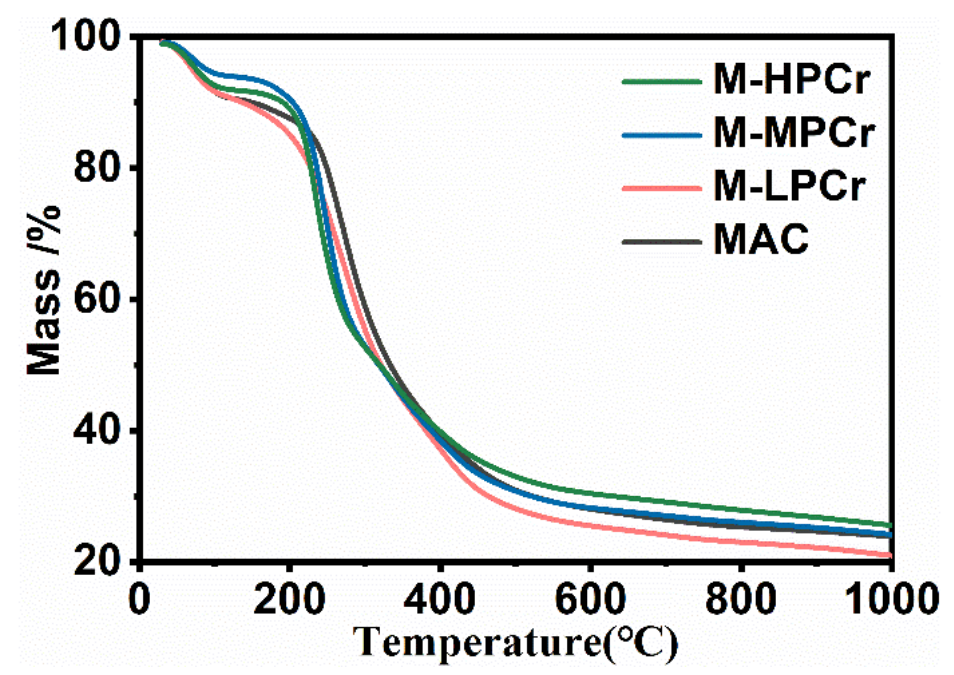

Figure S1. TGA results of MAC and MAC-PCr.

(a)

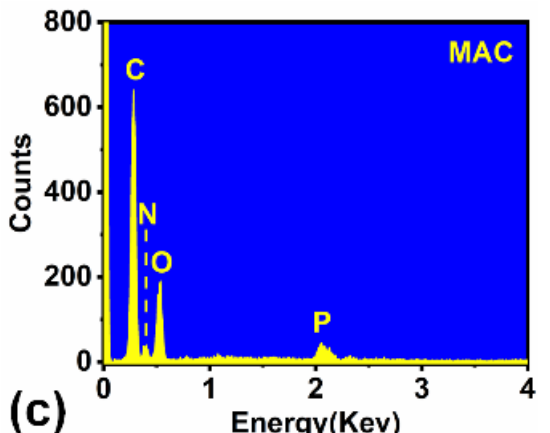

(c)

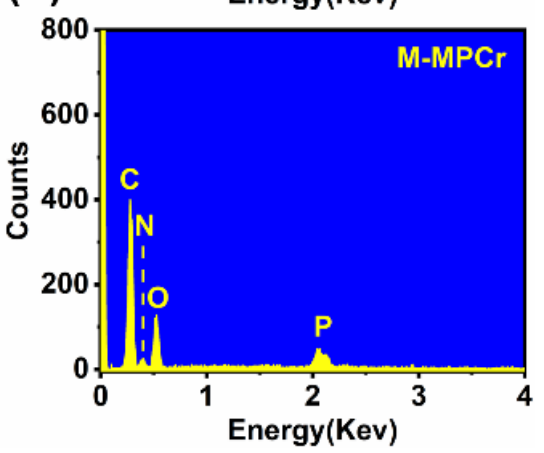

(b)
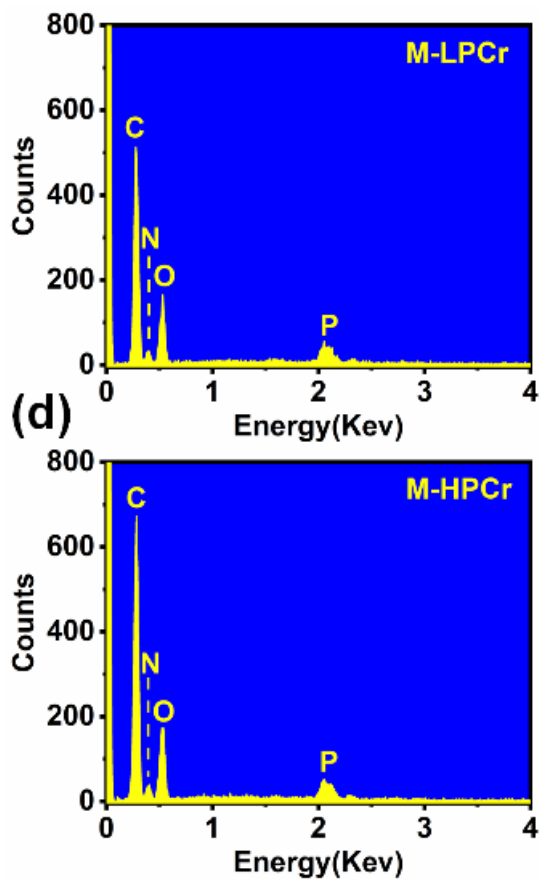

Figure S2. EDS results of MAC, M-LPCr, M-MPCr and M-HPCr hydrogels. 
Table S3. Elemental composition of MAC and MAC-PCr.

\begin{tabular}{cccccc}
\hline Materials & $\begin{array}{c}\text { Element } \\
\mathbf{C}(\%)\end{array}$ & $\begin{array}{c}\text { Element } \\
\mathbf{N}(\mathbf{\%})\end{array}$ & $\begin{array}{c}\text { Element } \\
\mathbf{O}(\mathbf{\%})\end{array}$ & $\begin{array}{c}\text { Element } \\
\mathbf{P}(\%)\end{array}$ & $\begin{array}{c}\text { Tatal } \\
\mathbf{( \% )}\end{array}$ \\
\hline MAC & 51.75 & 12.15 & 35.62 & 0.48 & 100 \\
M-LPCr & 51.74 & 12.04 & 35.38 & 0.83 & 100 \\
M-MPCr & 51.56 & 11.53 & 35.89 & 1.02 & 100 \\
M-HPCr & 62.01 & 7.48 & 28.45 & 2.05 & 100 \\
\hline
\end{tabular}
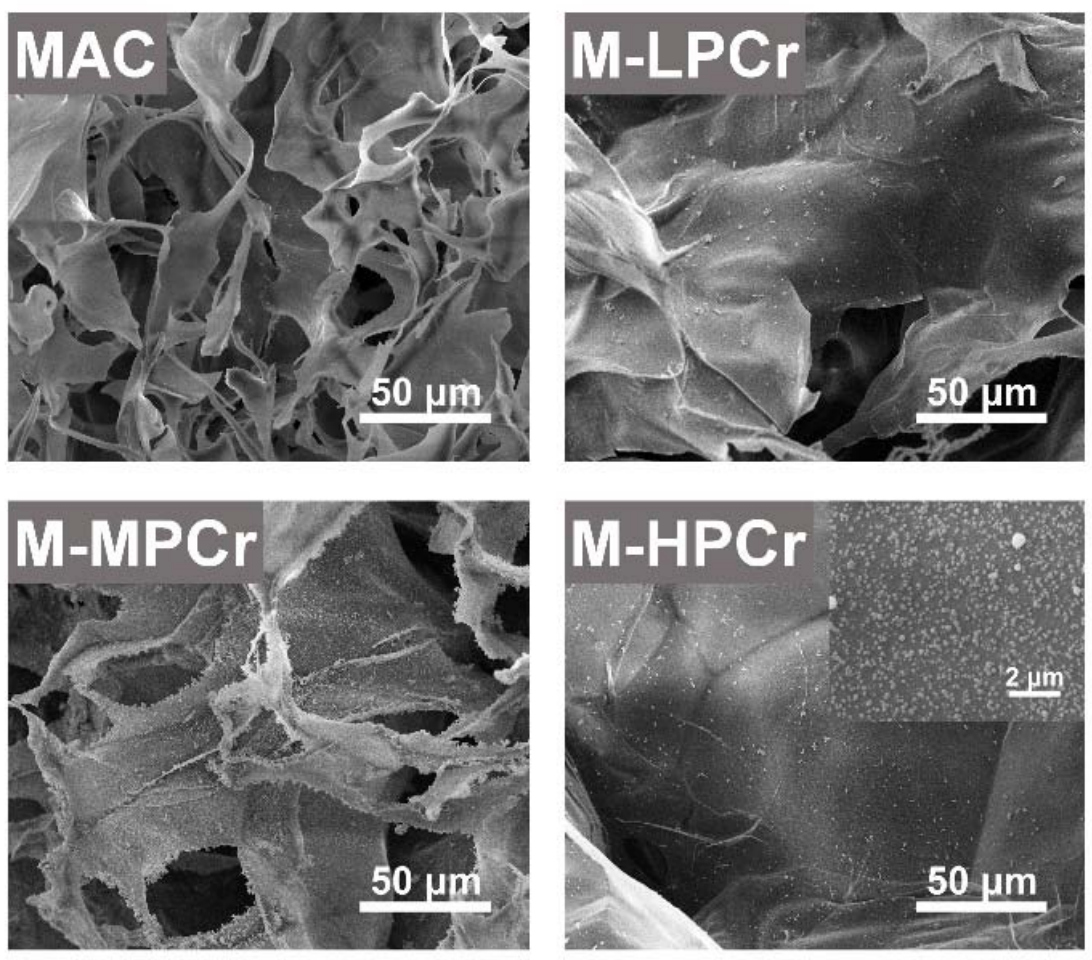

Figure S3. SEM images of MAC, M-LPCr, M-MPCr and M-HPCr hydrogels incubated in 5XSBF for $1 \mathrm{~h}$. 

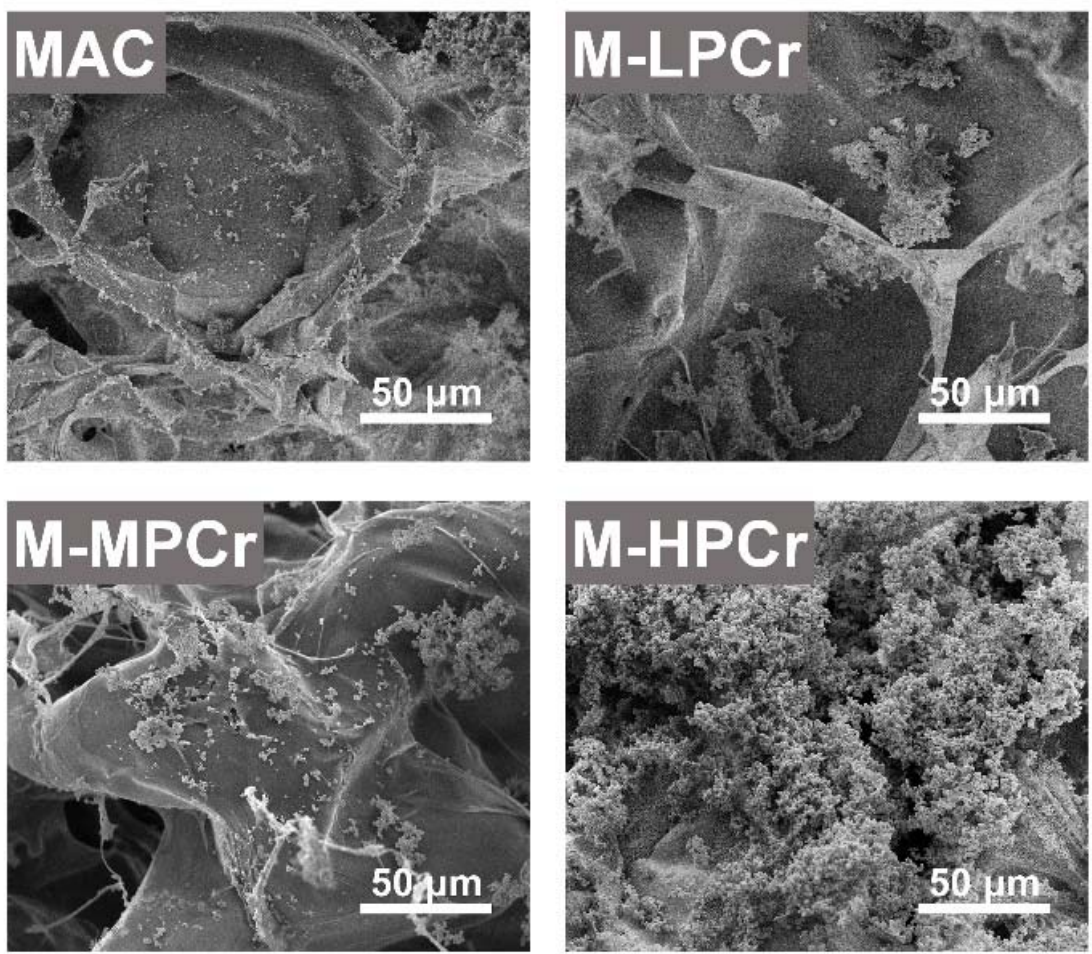

Figure S4. SEM images of MAC, M-LPCr, M-MPCr and M-HPCr hydrogels incubated in 5XSBF for $5 \mathrm{~h}$. 

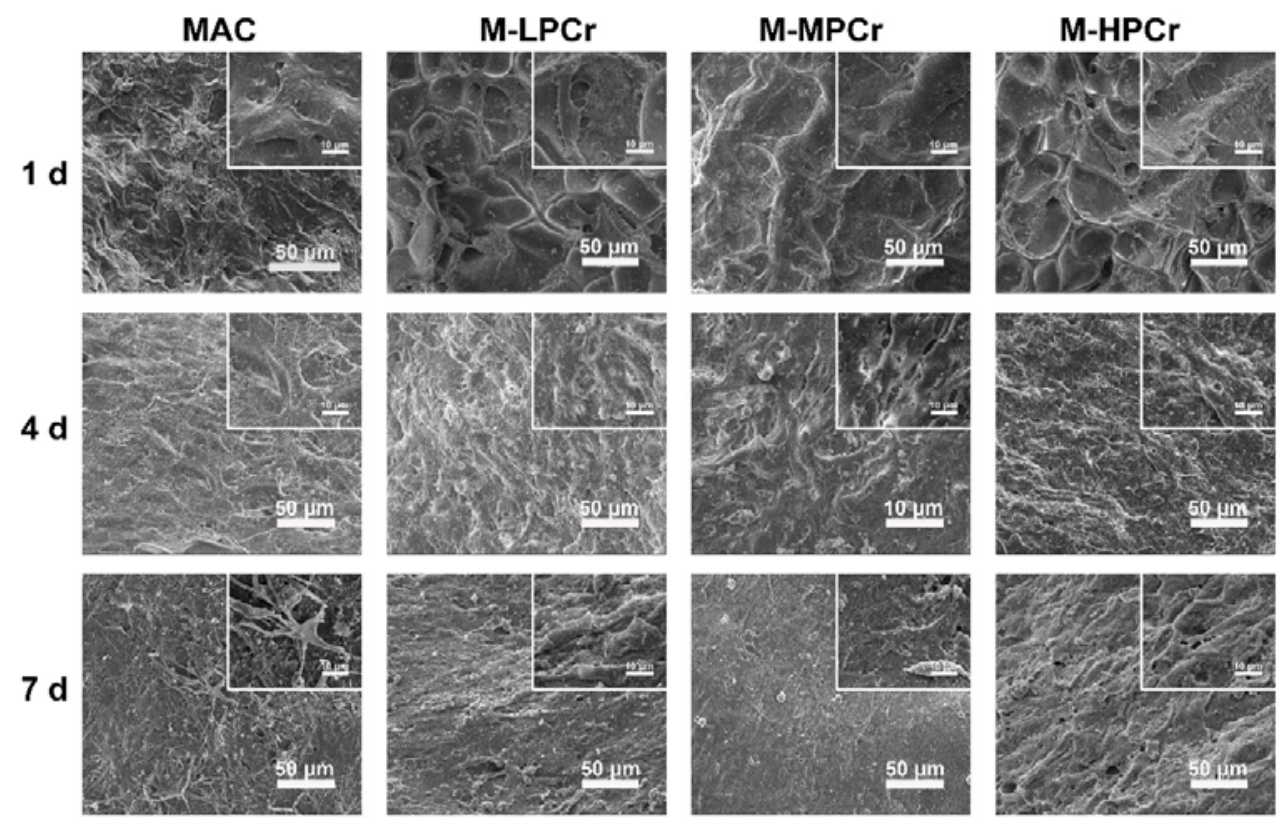

Figure S5. SEM images of hFOB cells cultured on hydrogels for 1, 4 and 7 days.
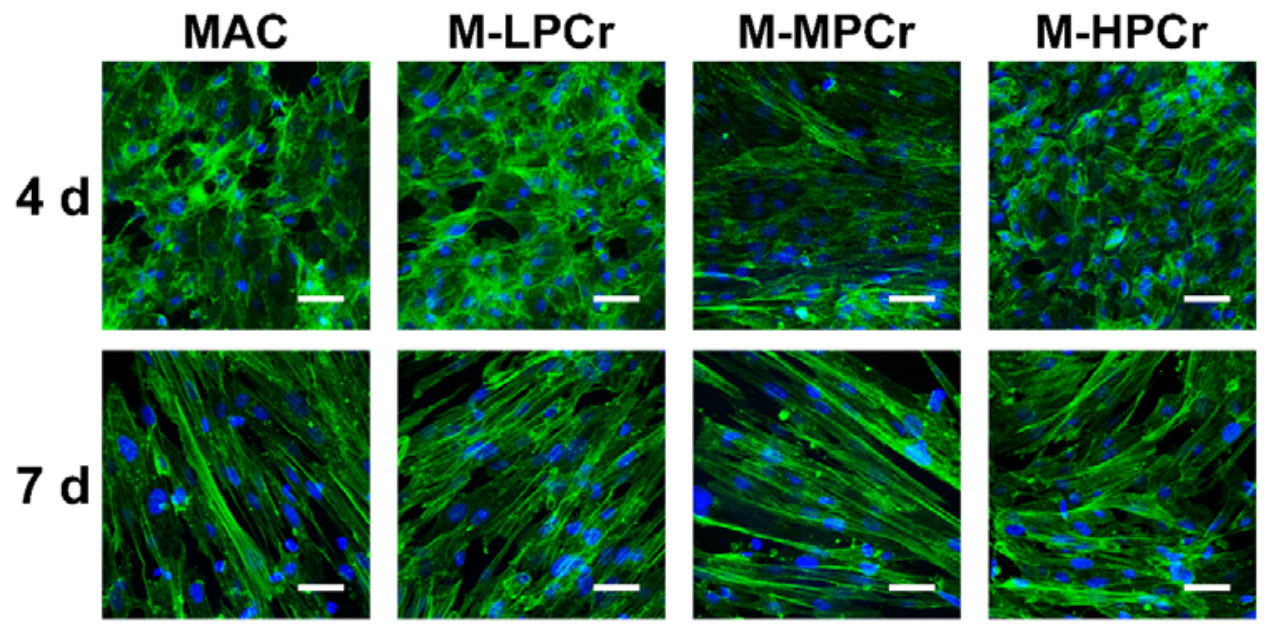

Figure S6. F-actin/Nucleus staining of hFOB cells cultured on hydrogels for 4 and 7 days. 

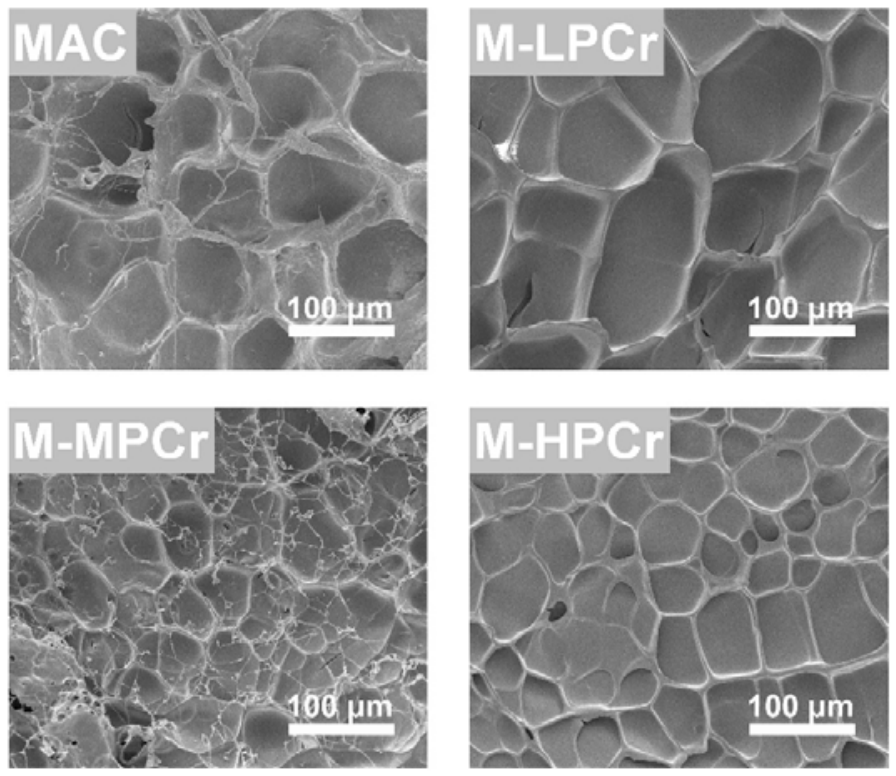

Figure S7. The surface microstructure of MAC, M-LPCr, M-MPCr and M-HPCr hydrogels after cells cultured for 7 days.
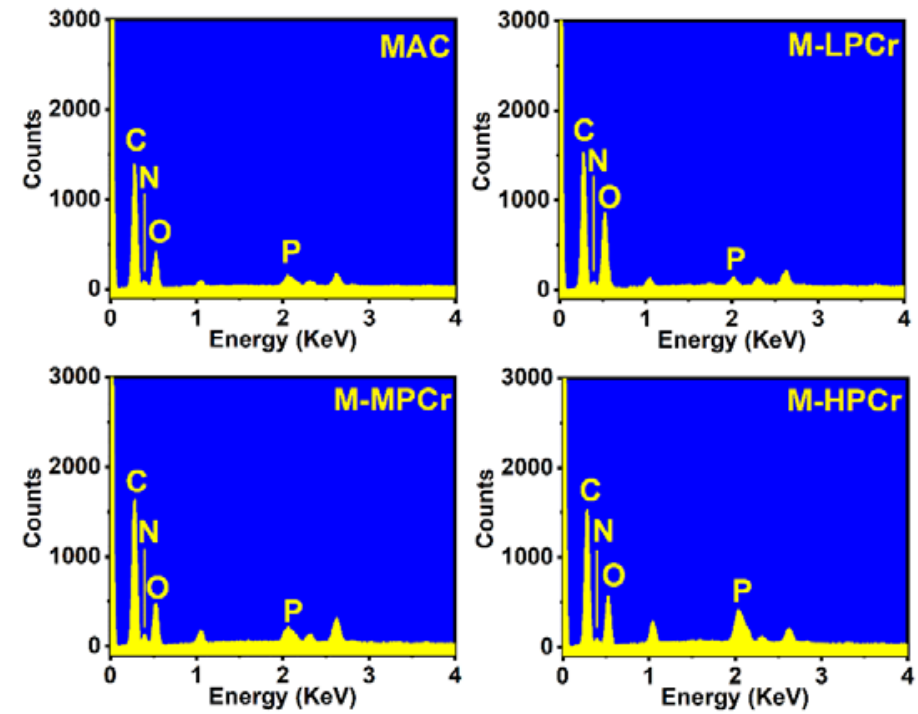

Figure S8. The EDS analysis of MAC, M-LPCr, M-MPCr and M-HPCr hydrogels after cells cultured for 7 days. 


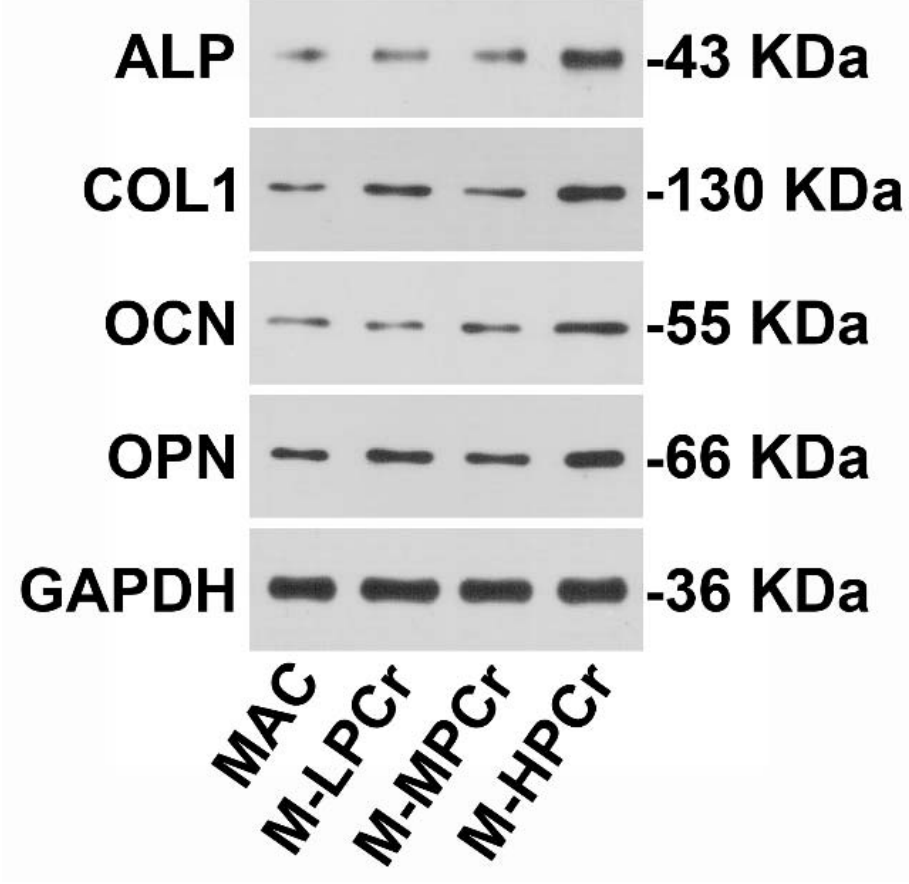

Figure S9. Western blot analysis of hFOB cells cultured on MAC, M-LPCr, M-MPCr and MHPCr hydrogels for 7 days.

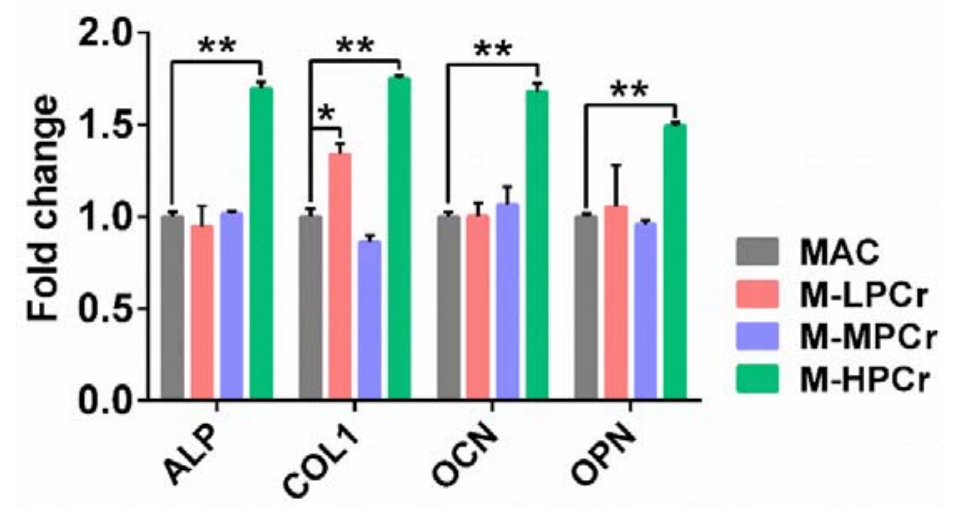

Figure S10. Summarized data showing the difference in protein expression of western blot results. 


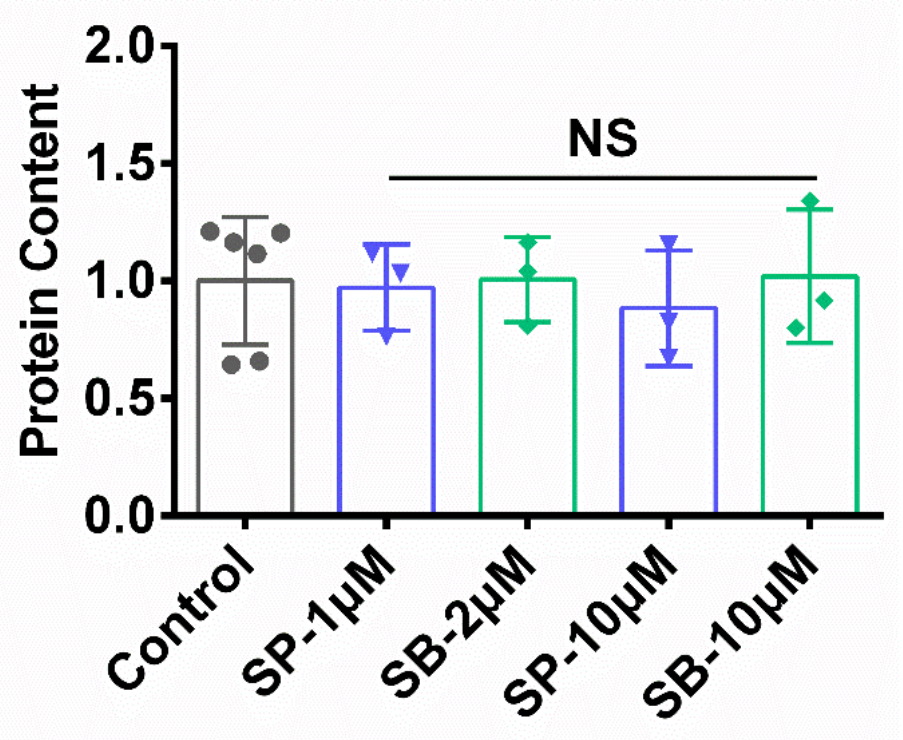

Figure S11. Protein content of hFOB cells cultured on MAC, M-LPCr, M-MPCr and M-HPCr hydrogels in normal medium, SP600125-added medium $(1 \mu \mathrm{M}$ and $10 \mu \mathrm{M})$ and SB203580-added medium $(2 \mu \mathrm{M}$ and $10 \mu \mathrm{M})$ for 7 days.

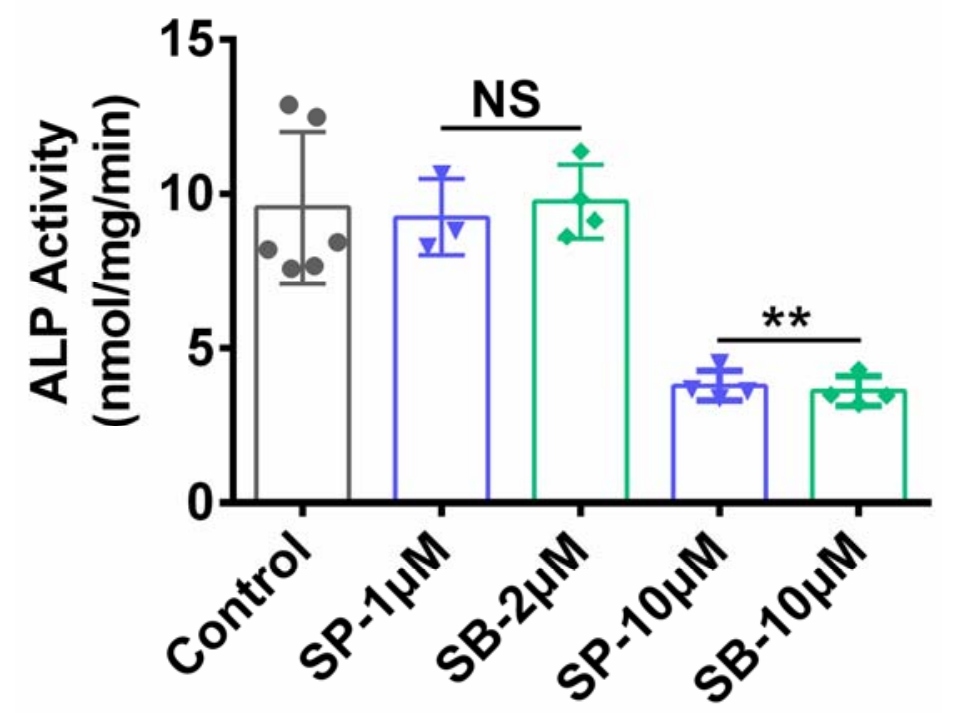

Figure S12. ALP activity of hFOB cells cultured on M-MPCr hydrogels in normal medium, SP600125-added medium ( 1 and $10 \mu \mathrm{M})$ and SB203580-added medium ( 2 and $10 \mu \mathrm{M})$ for 7 days. 